\title{
BIT LOADING FOR PRECODED DSL SYSTEMS
}

\author{
D. Zanatta Filho, R. R. Lopes, R. Ferrari, R. Suyama* \\ School of Electrical and Computer Engineering \\ University of Campinas - UNICAMP \\ \{daniloz,rlopes,rferrari,rsuyama\}@decom.fee.unicamp.br
}

\author{
B. Dortschy \\ Ericsson - ASP Lab
$\{$ boris.dortschy\}@ericsson.com
}

\begin{abstract}
Crosstalk is one of the main impairments of digital subscriber line (DSL) systems. To combat this impairment, several techniques were proposed in the literature that employ some form of precoding in the downstream. However, most existing bit allocation algorithms are not applicable to precoded DSL systems when the power in each transmission line is constrained. In this paper, we propose a bit allocation algorithm for this scenario. The proposed algorithm maximizes the bit rate given the available power. It somewhat extends single-user algorithms in that it sequentially allocates bit to the "best" user and tone. In the proposed algorithm we use a definition of "best" that is suitable for precoded systems. Simulation results show that the proposed algorithm achieves almost the same rates as those of power allocation algorithms, which do not take into account the discrete nature of the transmitted information.
\end{abstract}

Index Terms - Frequency division multiplexing, Multiaccess communication, Digital subscriber lines, Resource management, Crosstalk.

\section{INTRODUCTION}

Digital subscriber line (DSL) systems are widely used to provide broadband access to end users [1]. In these systems, data is transmitted through the copper pairs traditionally used only for telephony. By using the existing infrastructure to provide broadband access, DSL systems are an attractive and cost-effective solution to last-mile access.

The copper pairs used by DSL systems are usually deployed in binders, which may contain dozens of pairs. The proximity of the pairs results in electromagnetic coupling, so that signals from one line interfere with the others. This impairment is known as crosstalk, and is one of the main factors limiting the achievable data rates in DSL systems. Several crosstalk mitigating algorithms have been proposed [2,3]. These algorithms exploit the fact that most of the pairs in a binder originate from the same central office (CO). Thus, in

\footnotetext{
* This work was supported by the Research and Development Centre, Ericsson Telecomunicações S.A., Brazil.
}

the downstream, the $\mathrm{CO}$ may jointly process the signals from the corresponding users prior to transmission, aiming at mitigating crosstalk. This processing is called precoding, and its use implies that the signal transmitted in a given line is actually a combination of the signals from all the users.

Most DSL systems employ discrete multitone modulation (DMT), which divides the spectrum of each user in several independent tones [1]. In this case, determining how many bits each user should transmit in each tone is critical $[4,5]$. This bit allocation, also known as bit loading, must observe some power constraints: the power in each line cannot exceed the practical limitations of the amplifier that drives this line; the power transmitted in each line must observe a spectral mask imposed by the standards.

In a precoded system, when a bit is allocated to a given tone of a given user, the transmitted power on all lines at that tone is increased. In this paper, we propose an algorithm that takes this coupling between the lines into account. We will show that the resulting algorithm achieves rates close to those achieved by power allocation algorithms, which do not take into account the discrete nature of the transmitted bits.

\section{CHANNEL MODEL AND PROBLEM STATEMENT}

In this work, we assume a linearly precoded DSL system with a per-line power constraint, as shown in Figure 1. In this figure, the transmission is divided into several tones that do not interfere with one another. This is a consequence of the use of DMT by DSL systems [1]. In each tone, the channel is characterized by a matrix $\mathbf{H}_{k}$, where $\left[\mathbf{H}_{k}\right]_{i, j}$ represents the channel gain between transmitter $i$ and receiver $j$. Thus, the received vector on tone $k$ is $\mathbf{y}_{k}=\mathbf{H}_{k} \mathbf{x}_{k}+\mathbf{z}_{k}$, where $\mathbf{x}_{k}$ is the channel input vector and each element of $\mathbf{z}_{k}, z_{j}^{k}$, is an additive white Gaussian noise (AWGN), whose power is given by $\sigma_{j}^{k}$.

Figure 1 also shows the precoding matrix $\mathbf{M}_{k}$. As seen in this figure, the information signal from the $i$-th user at the $k$-th tone, $\tilde{x}_{i}^{k}$, goes through the precoding matrix $\mathbf{M}_{k}$ before generating the actual channel input. As a result, $x_{i}^{k}$ is a linear combination of the information symbols from all the users, i.e., $\mathbf{x}_{k}=\mathbf{M}_{k} \tilde{\mathbf{x}}_{k}$. The per-line power constraint is also illustrated in Figure 1, where it can be seen that the total transmit- 
ted power in a particular line must be less than $P_{\max }$.

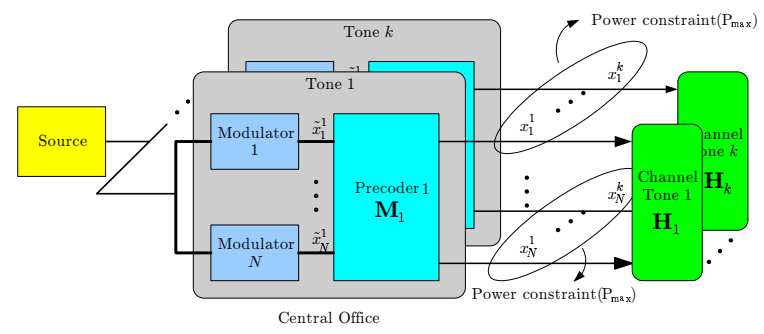

Fig. 1. Precoded DSL system.

In this paper, we assume that the precoder, along with any signal processing at the receiver, completely cancels the crosstalk. This can be achieved, for instance, with a zeroforcing (ZF) precoder, which was shown in [3] to yield nearoptimal rates. The goal of this paper is to propose a bit allocation algorithm for the system shown in Figure 1.

\section{EXISTING BIT ALLOCATION ALGORITHMS}

Several bit allocation algorithms have been proposed in the literature. Most of them are based on the gap approximation, pioneered by Forney [6]. Using this approximation, we may write the number of bits that can be transmitted on an AWGN channel as

$$
b=\log _{2}\left(1+\frac{S N R}{\Gamma}\right),
$$

where $S N R$ is the received signal-to-noise ratio (SNR) of the channel and $\Gamma$ is a parameter that depends on the type of constellation, target bit error rate (BER), the coding scheme used, and may allow for some margin. Equation (1) allows us to determine the energy needed to transmit a certain number of bits with a given BER in a DSL system. Indeed, the SNR of the $k$-th tone of the $i$-th user can be written as $S N R_{i}^{k}=\varepsilon_{i}^{k} \rho_{i}^{k}$, where $\varepsilon_{i}^{k}$ is the energy transmitted by the $i$-th user at the $k$-th tone and $\rho_{i}^{k}$ is defined as

$$
\rho_{i}^{k}=\frac{\left|g_{i}^{k}\right|^{2}}{\sigma_{i}^{k}},
$$

where $g_{i}^{k}$ is the channel seen by the $i$-th user at the $k$-th tone, taking the precoding into account. In other words,

$$
g_{i}^{k}=\left[\mathbf{H}_{k} \mathbf{M}_{k}\right]_{i, i} .
$$

Then, if we want the $i$-th user to transmit $b_{i}^{k}$ bits in his $k$-th tone with a given BER, the necessary energy is given by

$$
\varepsilon_{i}^{k}=\frac{\Gamma}{\rho_{i}^{k}}\left[2^{b_{i}^{k}}-1\right] .
$$

In a single-user system, the well-known Campello algorithm [4] provides optimal bit allocation. This algorithm is based on the idea of incremental allocation. Initially, zero bits are allocated to each tone. Then, at each iteration, the algorithm uses (4) to compute the incremental energy needed to transmit one more bit on the $k$-th tone. This incremental energy is defined as the difference between the energy that would need to be allocated to the $k$-th tone if it were to transmit one more bit and the energy currently allocated to this tone. The algorithm then allocates the bit to the tone requiring the least incremental energy. The iterations then repeat, with the algorithm allocating bits one at a time while the power constraints are observed.

However, in a multiuser DSL environment bit allocation becomes more involved. Indeed, assuming that no crosstalkmitigating algorithms are used, the SNR in (1) must be replaced by the signal-to-interference-plus-noise ratio (SINR), since in this case crosstalk is also treated as noise. Obviously, the SINR of a given line depends on the power of the remaining lines. Thus, if a bit is allocated to a given user, the power in the corresponding line must be increased, which decreases the SINR of the other users. If this decrease is not compensated for, the BER of the other users will increase.

Clearly, the problem of bit allocation in the multiuser case is coupled between the lines, and Campello algorithm is no longer applicable. In this case the optimum solution is unknown, and sub-optimal algorithms have been proposed in the literature. For instance, in [5] a bit loading algorithm based on the idea of incremental allocation is proposed. This algorithm computes the total power increase needed to allocate one bit to a certain user and tone, where the total power also takes into account the power increase needed to compensate for the additional crosstalk.

Another possible approach to the problem of bit allocation is to employ power allocation algorithms. After all, if the optimal power allocation is known, the number of bits can be determined using (1). The main difference is that, in power allocation, the fact that discrete bits will be transmitted is ignored, and it is assumed that the power in each line assumes continuous values. An integer number of bits may be obtained from the result of power allocation algorithms if, for instance, the latter is rounded down to the nearest integer.

The most traditional power allocation method is the waterfilling (WF) algorithm [3,7], which assumes that the signals are jointly processed at both ends of the communication link and that the total power to be transmitted, i.e., the sum of the powers of all lines involved, is limited. One drawback of waterfilling is that it does not take into account any constraint on the power transmitted through each line. Indeed, waterfilling employs a precoder that incurs no increase in the total transmitted power. However, depending on the channel, this precoding may result in a substantial increase of the transmitted power in one particular line.

Unfortunately, in practical systems where lines are driven by individual amplifiers, the power transmitted on each line must also be constrained. In [3], Cendrillon proposed a power 
allocation algorithm, called optimal power allocation (OPA), that takes this per-line constraint into account. A discrete bit loading algorithm obtained from rounding down the result of OPA is referred to as rounded-OPA (ROPA).

In summary, although several resource allocation algorithms were proposed for DSL systems, none of them solves the problems addressed by the algorithm proposed in this paper: to allocate bits (not power) to precoded systems with a constraint on the power transmitted in each line. In the next section, we present the proposed algorithm.

\section{BIT LOADING FOR PRECODED SYSTEMS}

In this section, we propose a bit loading algorithm for precoded DSL systems. The proposed algorithm is also based on incremental allocation, so it must compare the costs of allocating a bit to different users and tones. In the single-user case, this cost is a scalar, namely the power increase required by this bit allocation. However, in a precoded system, the signal transmitted in a line is a combination of the information symbols of all users. Thus, when a bit is allocated to a tone and a user, this actually represents an increase in the power transmitted on that particular tone in every copper pair involved. This results in a vector of power increase, and for incremental allocation we must determine which vector is better. Since it is not possible to sort vectors, in this paper we propose to compare the total power increase required by a bit allocation, i.e., the sum of the power increases needed by that allocation in all the pairs involved.

To describe the proposed algorithm, we need the following symbols:

- $b_{i}^{k}$ : Number of bits allocated to the $k$-th tone of the $i$-th user;

- $\varepsilon_{i}^{k}$ : Symbol energy associated with the $k$-th tone of the $i$-th user (before precoder matrix);

- $N_{\text {tones }}$ : Total number of tones per line;

- $N_{\text {lines }}:$ Total number of lines;

- $\mathbf{M}_{k}$ : Precoder matrix associated with the $k$-th tone;

- $p_{i}^{k}$ : Transmitted energy associated with the $k$-th tone of the $i$-th line (after precoder matrix) computed as:

$$
p_{i}^{k}=\sum_{m=1}^{N_{\text {lines }}}\left|\left[\mathbf{M}_{k}\right]_{i, m}\right|^{2} \varepsilon_{m}^{k} ;
$$

- $P_{i}^{\max }$ : Maximum power allowed in the $i$-th line.

The problem is then to maximize the total number of bits,

$$
\sum_{k=1}^{N_{\text {tones }}} \sum_{i=1}^{N_{\text {lines }}} b_{i}^{k}
$$

subject to a power constraint per line,

$$
\sum_{k=1}^{N_{\text {tones }}} p_{i}^{k} \leq P_{i}^{\max },
$$

for $1 \leq i \leq N_{\text {lines }}$.

The proposed algorithm is summarized in table 1 . We begin by allocating no bits to all tones and users. Then, we sequentially allocate bits to the user and tone that requires the least extra power. The algorithm stops when it cannot allocate another bit without violating the power constraints. It should be noted that other power constraints of practical importance can be easily incorporated into the algorithm. In the case of spectral masks, we may check if a given allocation requires more power in a given tone than allowed by the standard. For comparison purposes, we did not include such constraint, since other algorithms for multiuser systems do not take them into account.

Table 1. Proposed Bit Loading Algorithm

1. Initialization: Set $b_{i}^{k}=0$ for all $1 \leq k \leq N_{\text {tones }}$ and $1 \leq$ $i \leq N_{\text {lines }}$;

2. While $\sum_{k=1}^{N_{\text {tones }}} p_{i}^{k}<P_{i}^{\max }$ for any $i\left(1 \leq i \leq N_{\text {lines }}\right)$, i.e., while all constraints are satisfied:

(a) For $k=1, \ldots N_{\text {tones }}$ and $i=1, \ldots N_{\text {lines }}$, evaluate $\Delta P_{i}^{k}$, the energy increment associated to adding one bit to tone $k$ and user $i$

i. Let $\tilde{b}_{i}^{k}=b_{i}^{k}+1$;

ii. Evaluate the symbol energy $\tilde{\varepsilon}_{i}^{k}$ associated with $\tilde{b}_{i}^{k}$;

iii. Evaluate the transmitted power at tone $i, \tilde{p}_{i}^{k}$, for $1 \leq i \leq N_{\text {lines }}$, required by this new allocation;

iv. Compute the resulting total power increment;

$$
\Delta P_{i}^{k}=\sum_{l=1}^{N_{l i n e s}}\left(\tilde{p}_{l}^{k}-p_{l}^{k}\right)
$$

(b) Find $k^{*}$ and $i^{*}$ such that $\left(k^{*}, i^{*}\right)=\arg \min \Delta P_{i}^{k}$, and still respect the per-line power constraint;

(c) Add one bit from the optimum position: $b_{i^{*}}^{k^{*}}=b_{i^{*}}^{k^{*}}+1$;

(d) Compute $\varepsilon_{i}^{k}$ and $p_{i}^{k}$ for this new bit allocation;

3. End.

\section{RESULTS}

The performance of the proposed bit loading algorithm was assessed through simulations, wherein the rate as a function of the reach was evaluated for a 10-pair MIMO-VDSL channel generated using Bin Lee's channel model [7]. The wire diameter is $0.5 \mathrm{~mm}$ (24-AWG). As per the VDSL standard [8], we used the downstream tones specified in the band plan A (previously known as 998), with tone spacing $\Delta f$ equal to $4.3125 \mathrm{kHz}$ and the DMT symbol rate $f_{s}$ equal to 4 $\mathrm{kHz}$. Background noise is composed by white thermal noise 
with a PSD of $-140 \mathrm{dBm} / \mathrm{Hz}$ and the available transmitted power for each line is $+11 \mathrm{dBm}$. Our computations are based on the gap approximation: the target BER is $10^{-7}$ or less, the coding gain was set to $3.8 \mathrm{~dB}$ and the noise margin to 6 $\mathrm{dB}$, which leads to a gap of $\Gamma=9.8-3.8+6=12 \mathrm{~dB}$. The proposed algorithm, OPA and ROPA use a ZF precoder [3].

The bit rates achieved by the proposed algorithm for the binder in the downstream for several channel lengths are shown in table 2. The rates achieved by other algorithms mentioned in section 3 are also shown. As seen in this table, the two power-allocation algorithms (OPA and WF) achieve the best performance. However, the proposed algorithm is the best among the bit allocation algorithms. In fact, it outperforms rounded OPA, the second best bit allocation algorithm, by around $30 \sim 50 \mathrm{Mbps}$. On the other hand, the proposed algorithm achieves a rate close to that of OPA, incurring a rate loss of at most $0.42 \%$. Note, however, that OPA computes power allocation, and may yield a non-integer bit allocation.

Table 2 highlights some interesting facts about DSL systems. First, the algorithms that employ precoding achieve rates much higher than Lee's algorithm, which does not perform any crosstalk mitigation. Thus, it is clear that crosstalk is indeed one of the limiting factors in the performance of DSL systems. Also, OPA and waterfilling achieve similar rates. Thus, as claimed in [3], ZF precoding does incur negligible capacity loss in DSL systems.

Table 2. Downstream Rate (Gbps) vs. Reach(m)

\begin{tabular}{|c|c|c|c|c|}
\hline Reach & 150 & 450 & 750 & 900 \\
\hline WF & 1.9526 & 1.1809 & 0.6233 & 0.4910 \\
\hline OPA & 1.9526 & 1.1807 & 0.6213 & 0.4906 \\
\hline Proposed & 1.9496 & 1.1770 & 0.6188 & 0.4885 \\
\hline ROPA & 1.8952 & 1.1217 & 0.5817 & 0.4577 \\
\hline Lee [5] & 1.3051 & 0.7858 & 0.4449 & 0.3565 \\
\hline
\end{tabular}

\section{CONCLUSION}

This paper presented a novel algorithm that allocates bits to the tones precoded DSL systems based on DMT. Precoding mitigates one of the main factors limiting the rates achievable by DSL systems: crosstalk. The proposed algorithm allocates an integer number of bits to each tone of each line, while limiting the power on each line to a specific power cap. Other power constraints, such as spectral masks, can be easily incorporated into the algorithm.

Simulation results show that the proposed algorithm achieves a data rate close to that of OPA, which is the best rate achievable by the system when the power on each line is constrained. Note, however, that OPA ignores the fact that the number of bits in each tone is discrete, so that it may result in a non-integer bit allocation, which restricts its practical usefulness. Thus, the proposed method achieves a performance close to optimal while observing the practical constraint of integer bit allocation.

The proposed algorithm also compares favourably to ROPA, an alternative bit allocation method for precoded DSL systems. In fact, the proposed algorithm obtains rates that are $30 \sim 50$ Mbps higher than those achieved by ROPA.

\section{Acknowledgments}

Some of the authors want to acknowledge the financial support received from the European Commission IST 6th framework and from the Swedish Agency for Innovation Systems, VINNOVA, through the IST - MUSE and the Eureka - Celtic BANITS projects respectively, which partially enabled this work. The authors would also like to thank Glauco Simões, Cristina Wada and Murilo Loiola for the discussions and help with the simulations.

\section{REFERENCES}

[1] T. Starr, J. M. Cioffi, and P. J. Silverman, Understanding Digital Subscriber Line Technology, Prentice Hall Communications Engineering and Emerging Technologies Series. Prentice Hall, 1999.

[2] G. Ginis and J. M. Cioffi, "Vectored transmission for digital subscriber line systems," Journal on Selected Areas in Communications, vol. 20, no. 5, pp. 1085-1104, June 2002.

[3] R. Cendrillon, Multi-User Signal and Spectra Coordination for Digital Subscriber Lines, Ph.D. thesis, Katholieke Universiteit Leuven, Leuven, Belgium, December 2004.

[4] J. Campello, "Practical Bit Loading for DMT," in Proc. IEEE International Conference on Communications (ICC'99), Vancouver, Canada, June 1999, pp. 801805.

[5] J. Lee, R. V. Sonalkar, and J. M. Cioffi, "Multiuser Bit Loading for Multicarrier Systems," IEEE Transactions on Communications, vol. 54, no. 7, pp. 1170-1174, July 2006.

[6] G. D. Forney and G. Ungerboeck, "Modulation and coding for linear Gaussian channels," IEEE Transactions on Information Theory, vol. 44, no. 6, pp. 2384-2415, October 1998 .

[7] B. Lee, Binder MIMO Channels, Ph.D. thesis, Stanford University, Stanford, USA, November 2004.

[8] ITU-T, G.993.1 VDSL (Very High Speed Digital Subscriber Line Transceivers), International Telecommunication Union, 2004. 